\title{
Application of Wavelet De-noising Technique on a Congested Internet Protocol (IP) Network
}

\author{
Danladi Ali \\ Department of Information Technology and \\ System, \\ National Metallurgical Academy, Ukraine
}

\author{
Abdullahi M. \\ Department of Laboratory Technology, Mudibbo \\ Adamawa University, Yola. Adamawa State Nigeria
}

\begin{abstract}
In this work, a model of a corporate network has been developed, simulated and implemented using optimized network engineering tool technology in a simulation environment of $100 \mathrm{~m} \times 100 \mathrm{~m}$ office network topology. We monitored delay traffic, used one dimensional multilevel wavelet de-noising technique to filter the possible cause of the network congestion and used ACF and FFT to validate the result of the de-noising after all the stages of decomposition and reconstruction processes. The result of the filtering process revealed that error in the data is de-noised successfully as the coefficient of ACF grows above zero and the energy rate in the FFT- spectrum increased.
\end{abstract}

Keywords: Wavelet, delay, decomposition, reconstruction, technique, ACF and FFT.

\section{INTRODUCTION}

Nowadays, the demand for internet service is growing exponentially more especially with the introduction of multimedia services such as video and audio streaming in addition to the traditional WWW browsing, e-mail and so on over IP network [10]. Due to these massive activities imposed on the internet network the data traffic is becoming more complex day by day. Hence, the internet network experience congestion as well as delay phenomenon. When viewing the causes of network congestion on a large scale many factors could be put into consideration; like low bandwidth, application of many hosts in a broadcast domain, multicasting and inefficient protocols. Congestion degrades overall network performance since it is associated with amplified data delay, data rate loss, retransmission of data and probably may involve data drop [5]. Congestion problem is very difficult to predict because one may not know the exact input characteristics of the network at a particular period of time. Therefore, in trying to address the congestion problem on the internet network and to develop an algorithm for the fast flow of data traffic across the IP network question of constructing a model with input characteristics arises. Many research works in this field have justified that recent IP network traffic suffers congestion problems. In [9], a study on internet delay was carried in order to analyze delay and causes of delay across the internet. The study shows that internet network exhibit delay caused due to inefficient design of a webpage. In [6], UDP echo method was used to investigate network problems as a faulty network interface card; the study revealed that, packets are lost randomly. In [2] the study was based on loss and delay measurement in data collected from internet service providers (IPS) in US the study arrived at the conclusion that some paths experienced severe impairments due to network protocol, failure and reconfiguration, and router operation may be responsible for network delay instead of normal congestion and quality of service. In [7], research was conducted to study network efficiency in terms of measuring network congestion; the result of the work revealed that most congestion problems were experienced due to the network components which include structural failure, links, nodes, other such as a natural disaster, and attack from terrorist. According to [3] "there exist stability in resulting delay difference in the path before and after the routing events", A work conducted on two methods of wavelet shrinkage, biased on high frequency removal shows that, wavelet tool is good a tool for filtering high frequency, see [13]. In [15], the work was conducted on filtering of unwanted signal from non-stationary bowel sound signal; the work reveals that the wavelet tool is capable of improving the signal to noise ratio of the non-stationary bowel sound. Another study carried on denoising ECG signal by discrete wavelet transform confirmed that; wavelet is an appropriate tool for de-noising the signal and improving signal to noise ratio [16].

In this work, we propose to develop an Ethernet network using OPNET technology with complex input, say 200 host users, monitor the delay traffic and to de-noise the probable noise in the delay traffic by applying 1D wavelet de-noising technique. There are many methods in which congestion and delay can be reduced or address; among all are segmenting the network using some equipment like switch, bridge, routers, using high bandwidth [14], and suitable protocols, but this work choose wavelet de-noising technique because, it seems to be one of the best and promising methods in de-noising congestion and delay problems in the entire network. To ascertain that de-noising application is effective, we will emphasize on the properties of autocorrelation function (ACF) in terms of a strong relationship with delay and fast Fourier energy spectrum to validate our results. One obvious observation with $\mathrm{ACF}$ is that as the presence of congestion or delay in the network increases its coefficient to decay to near zero [11] and in the fast Fourier transform (FFT) energy spectrum; the energy rate in the spectrum reduces while the traffic volume transmission reduced to a considerable extent. This is the major reason of choosing the wavelet technique. However, in [15][16], The level at which the filtering effect is effective is not ascertain in terms of accuracy. But, in this work, the use of ACF and FFT energy spectrum confirmed the effectiveness of the filtering.

\section{IMPLEMENTATION OF THE SIMULATION MODEL}

A simulation area of $1000 \mathrm{~m} \times 1000 \mathrm{~m}$ office topology network is first created using a startup wizard. The topology is then created. The required numbers of the nodes are dragged into the empty space base on the number of nodes needed for a 
particular department, and then each of the node's fields is set to carry out their functions. These include application configuration, profile configuration, server, switch, person computer and LANs. The Table 1 below gives the model and the simulation metric parameters.

Table I. Simulation Parameters

\begin{tabular}{|l|l|}
\hline Simulation Nodes & $\begin{array}{l}\text { Simulation } \\
\text { parameters }\end{array}$ \\
\hline Simulation size & $100 \mathrm{~m} \times 100 \mathrm{~m}$ \\
\hline Traffic monitored & $\begin{array}{l}\text { HTTP, FTP, Telnet Session, } \\
\text { E- Mail, Database }\end{array}$ \\
\hline Simulation time & $1800 \mathrm{~s}$ \\
\hline App. configuration setting & Default \\
\hline 2 LAN & 50 and 100 hosts users \\
\hline Information department & 14 host users \\
\hline Technical department & 6 host users \\
\hline Administrative department & 30 host users \\
\hline Total hosts & 200 host Users \\
\hline
\end{tabular}

\subsection{Network Design}

There are typically three (3) classes of IPv4 network address that is class; A, B, C. Class A support a subnet mask of 255.0.0.0, B support 255.255.0.0 and C support 255.255.255.0. To calculate the number of hosts for each department, we can use the expression (1)

$$
N_{h}=2^{n}-2
$$

Where $N_{h}$, is the total number of hosts in each department, $\mathrm{n}$ is the number of zero bits in the subnet mask. But in this work, there are 200 host users. Therefore, class $\mathrm{C}$ is more suitable since it accommodates up to 254 host users. Considering the fact that this network consists of two LANs, the network address has to be divided into two and assuming that the 50 host users are on the same network with the administrative, and technical department making a total of 86 host users on one network, while the 100 host users and the information department are on the second network making a total of 114 host users. Moreover, the meaningful IP address of each network will be 192.168.1.0-85/24 or 192.168.1.0-85 255.255.255.0 and second network the IP address will be 192.168.2.0-113/24 or 192.168.2.0-113 255.225.255.0

\subsection{Collection of Statistics}

A large amount of data traffic from different sources is introduced into the model. The goal is to monitor delay with the huge data traffic. In optimized network engineering tool there are two major statistics available, these are; global statistics and node statistics. Global statistics tell us about the statistics of the entire network while node statistics tells us about the statistics of an individual node. Appropriate statistics are then imposed on the scenario model. Simulation is run and the results are taken, the monitored delay data traffic is presented in Fig 1 below as a signal.

\section{RESULTS AND DISCUSSION}

Fig 1, depicted below is the Ethernet delay (data traffic) due to congestion. This data traffic is collected from the OPNET simulator, as we can see the data traffic varies with time, the inconsistency may attribute to the variation of the input characteristics of the data traffic at the time of the day that shows stationary behavior with time.

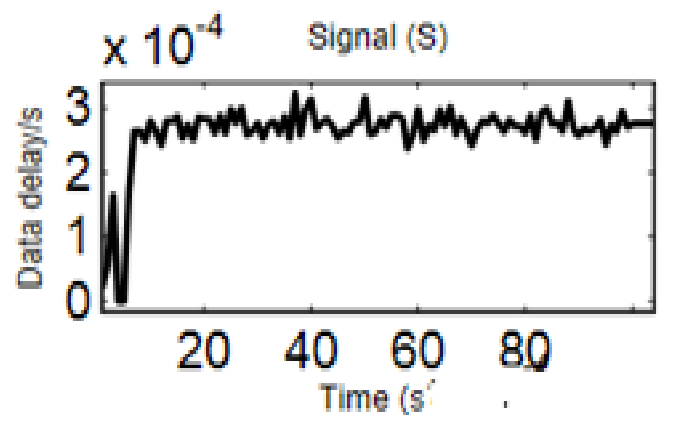

Fig 1: Delay traffic monitored

While Figure 2, presents the histogram of the delay data traffic showing the greatest delay concentration towards the later part of the signal which means at that point the signal has a high frequency and may be undesirable for the network.

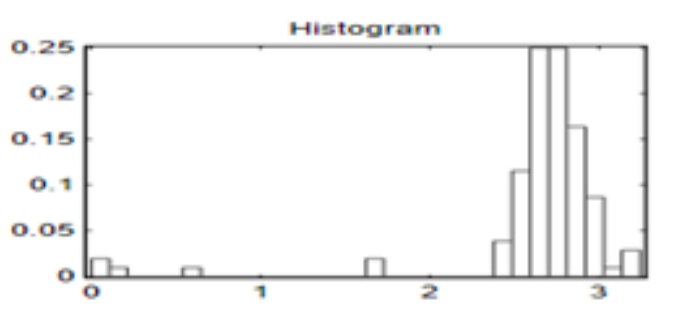

Fig 2: Histogram of the data delay monitored

As mentioned in the previous part of this text, congestion or delay is undesirable for any network user. However, it becomes necessary to monitor our network in order to provide a reasonable quality of service $(\mathrm{QoS})$ to the end user. This is one of the factors that prompt us to use the wavelet technique as one of the promising methods suitable for decongesting cause of the delay in the network. The wavelet technique utilizes decomposition, compressing and reconstruction process before the signal is de-noised [8][12][13]. The process of decomposition consists of down sampling and filtering while reconstruction process involve up sampling and filtering. The decomposed signal gives information about the signal as given in (2)

$$
y=a_{x}(J, k) \psi_{0}(J, k)+\sum_{k=1}^{j} d_{x}(j, k) \psi_{0}(j, k)(2)
$$

The coefficients are situated on the upper and lower scale on the octave ranging [17] from $1 \leq \mathrm{J} \leq \mathrm{Jn}$, and $1 \leq \mathrm{j} \leq \mathrm{Jn}$ for the approximated and the detail signal. The wavelet coefficients $a_{x}(J, k), \quad d_{x}(j, k)$ are derived from the relationship below after complete decomposition.

$$
\psi_{a, b}(t)=\frac{1}{\sqrt{a}} \psi\left(\frac{t-b}{a}\right)
$$

Where $a$ is the positive number which defines the scales and $b$ is the real number that defines the shifts in (3) sometimes called child wavelets derived from the mother wavelet. The decomposition and the reconstruction procedures are shown in 
the Fig 3.

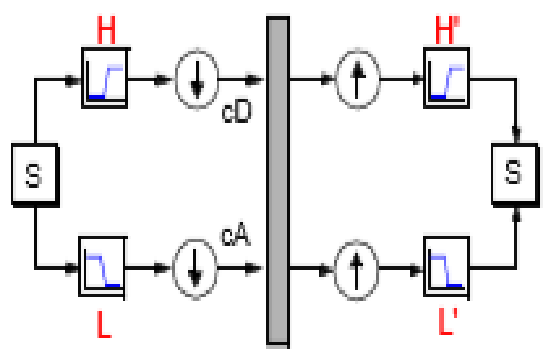

Decomposition Reconstruction

\section{Fig 3: Full decomposition and reconstruction process}

During the process of the filtering in any of the stage attention must be given to the choice of the appropriate filter because if care is not taking vital part of the information may be lost since the signal always consists of high and low frequencies. We transfer the Ethernet delay data traffic from the OPNET simulator to the Simulink in the MATLAB for analysis. The signal is then analyzed with wavelet db4 type at level 3, decomposition with interest of de-noising the undesirable content of the signal that leads to congestion or the delay. Before the process of decomposition and reconstruction it is expected that the delay data traffic contains high noise level, when looking at it from theoretical and traffic engineering view [1], the signal will have a strong autocorrelation function; decaying to zero if a large amount of noise exists in the signal content and the fast Fourier transform energy spectrum; the energy rate will reduce due to delay while the volume of the successful traffic that will be transmitted to the destination will also be reduced. In this work, we identified the presence of the delay in the signal by decomposing and reconstructing the signal while closely observing the behavior of the ACF and FFT of the signal at each stage of decomposition and after the reconstruction process as shown in Fig 6 and 7. Fig 4, shows the reconstructed signal after all the stages of decomposition and filtering. Comparing Fig 1 and 4, we can see that some parts of the signal in Fig 1, have been smoothed meaning the signal is de-noised which evident that Fig 1 contained some reasonable amount of noise though both the signal follows the same trend.

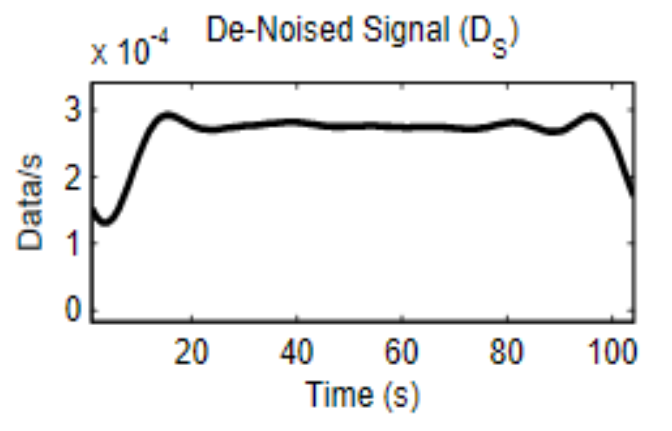

Fig 4: Reconstructed signal

Fig 5, shows the residual signal; this is an undesirable or the signal content filtered from Fig 1, it consumes almost all the content of the signal in Fig 1. This is evidence that the larger portion of the original signal is occupied by a noise and it may attribute to the cause of the congestion on the network.

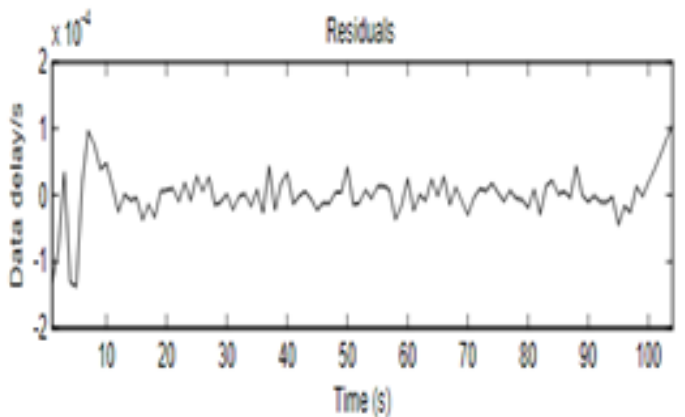

Fig 5: Residual signal after de-noising

Fig 6 and 7 show the fast Fourier transform spectrum energy spectrum and autocorrelation function respectively. During the de-noising process at each level, we noticed that coefficient of ACF increases to above zero and the FFT energy shows more traffic flow; here when comparing the $\mathrm{ACF}$ and the original signal after decomposition and the reconstruction of the signal shows poor correlation which means the network is de-noised to a reasonable extend since ACF characteristics does not comply, in terms of the relationship with delay while looking at the FFT spectrum in Fig 6 indicates that traffic flow rate is tremendous due the de-noised process compare the traffic flow rate in Fig 1.

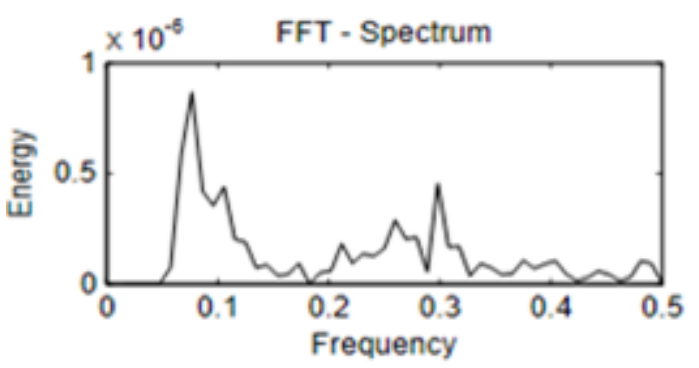

Fig 6: FFT-Energy spectrum after de-noising

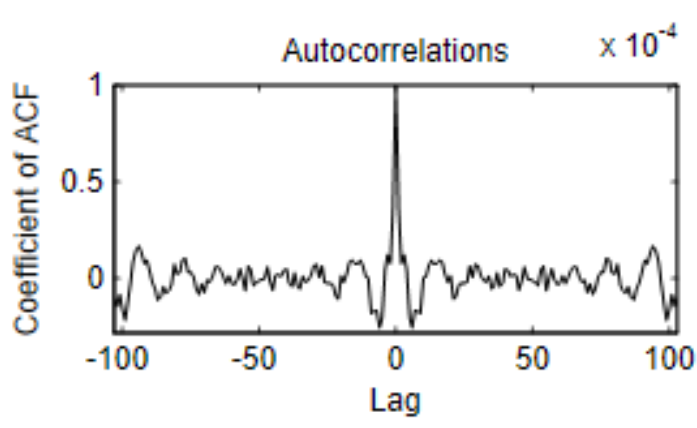

Fig 7: Autocorrelation function after de-noising

\section{CONCLUSION}

A corporate network had been developed, simulated and implemented to monitor data delay. Simulation result shows that the corporate network experience high delay due the high input characteristics of the network. The delay data is monitored and de-noised through 1D wavelet technique. After application of the de-noising technique ACF and FFT, confirmed that the noise content of network had been successfully de-noised. This work is focused on the monitoring of delay data or congestion and de-noising the process in the corporate network but does not take in 
consideration the causes of the network congestion. We believed that this work has provided some vital information on how de-noised signal from congested network using wavelet technique. It is recommended that such work should use ACF and FFT to relate the strong affinity between delay and long range dependence or self-similarity.

\section{REFERENCES}

[1] P. Abry and D. Veitch, "Wavelet analysis of long range dependence traffic", IEEE Transactions on information theory, 1998.

[2] M. Athiama, T. Fouad, and K. Mansouross and delay measurement of internet backbone. Computer communication special issue on monitoring and measurement, 2005

[3] P. Hamibindu, Z. Ying, Z. M. Morley and Y. H. Charlie. "Understanding network delay changes by routing events" Proceeding of ACM SIGMETRICS, 2007.

[4] D. Hayes and G. Armitage, "Improving co-existence and loss tolerance for delay-based TCP congestion control" Proceeding of IEEE LCN, 2010, pp. 24-31

[5] R. Jain, "A delay - based approach for congestion avoidance in interconnected Heterogeneous computer network", ACMSIGCOMM computer communication review vol. 19 no. 5,1989 , pp. 56-71

[6] C. B. Jean., "End to end packet delay and loss behavior in the internet" ,SIGCOMM's-Ithaca, N.Y US.,1993.

[7] A. Nagurney, Q. Qiang, “A efficiency measure for congested network", Epl Journal, 2007.

[8] J. L. Passmore, B. C. Collings, and J. P.Collings, "Autocorrelation "of electrical noise", Journal Physics 63,
1999, pp. 592-596

[9] G. Pradeep, "Study on internet delays" Masters Theses department of computing science, Umea Sweden, 2005.

[10] A. Qazi, L. L. H Andrew, and T. Znati, "Congestion control with multi packet feedback", IEEE/ACN transaction, on networking vol. 20 no. 6, 2010, pp. 17211733.

[11] P. Quan, Z. Lei, D. Guanzahang, and Z. Hongcai , "Two de-noising methods by wavelet tranform", IEEE transaction of signal processing vol. 47 no. 12, 1999, pp. 3406-3409

[12] R. Q. Quian and H Garcia, "Single-trial event- related potential with wavelet de-noising", Clinical Neuro physiology 2003, pp. 76-390

[13] C. Rami, "Signal de - noising using wavelets", Project report. 2011.

[14] D. A. Shalangwa. Evaluation of bandwidth performance in a Corporate network", Academic Journal of engineering and Technology Research vol. 6 no. 1, 2014, pp. 1-5.

[15] Z. Xizheng, R. Yuanqing, W. Weixiong, A new filtering methods in the wavelet domain for bowel sound", International Journal of advance computer science and applications, vol. 1 no. 5, November, 2010.

[16] A. Aouinet, and C. Adnane, "Electrocadiogram de-noised signal by discrete and continuous wavelet transform signal processing international journal, vol. 8 no. 1, 2014, pp. 19.

[17] Danladi Ali and Vlada N. Y. Wavelet based path loss modeling for global system for mobile communication in an urban environment International Journal of Science and Research 3(7): 1929-1932 